\title{
Comparison of the effects of argan and nigella oils on malathion-induced cognitive-behavioral alterations and brain histopathology in male Wistar rats
}

\author{
Pacôme Kouadio N'Go ${ }^{1,3, *}$, Emmanuel Diboh ${ }^{2}$, Idrissa Sylla ${ }^{2}$, Ahmed Omar Touhami Ahami ${ }^{3}$, \\ Youssef Aboussaleh $^{3}$, Fatima-Zarha Azzaoui ${ }^{3}$ and Samira Boulbaroud ${ }^{4}$ \\ ${ }^{1}$ Peleforo Gon Coulibaly University, Training and Research Unit of Biological Sciences, Korhogo, \\ Ivory Coast; ${ }^{2}$ Jean Lorougnon Guede University, Training and Research Unit of Environment, \\ Daloa, Ivory Coast. ${ }^{3}$ Unit of Clinic and Cognitive Neurosciences. Laboratory of Biology and Health. \\ Department of Biology, Faculty of Sciences Kenitra, University Ibn Tofail; ${ }^{4}$ Polydisciplinary Faculty, \\ Laboratory of Biotechnology and Sustainable Development of Natural Resources, \\ Sultan Moulay Ismail University, Beni Mellal, Morocco.
}

\begin{abstract}
Occupational or environmental exposure to malathion is known to increasingly cause cognitive or psychiatric disorders, since it damages brain tissue through inflammation and oxidative stress processes. Here, we examine the possible neuroprotective effects of some natural products such as argan and nigella oils against malathioninduced cognitive-behavioral deficits and cerebral tissue alterations. Male Wistar rats were used in the present study. The rats were pretreated either with virgin argan oil ( $2 \mathrm{ml} / \mathrm{kg}$ p.o.) or nigella oil (1 ml/kg p.o.) 1 hour before malathion (150 $\mathrm{mg} / \mathrm{kg}$ p.o.) administration for 28 days. At the end of the experiment, the cognitive-behavioral tests were performed, followed by the biochemical and histological analysis. Our results confirmed significant recognition memory deficits, affective disorders such as anxiety-like behavior in malathion-exposed rats, as well as an increased acetylcholinesterase (AChE) inhibition rate and histological changes in the brain. However, the pretreatment with argan oil significantly reversed the memory and behavioral disorders, and prevented neurodegeneration in the hippocampus and prefrontal cortex (PFC), caused
\end{abstract}

\footnotetext{
*Corresponding author: ngo.pacome@upgc.edu.ci
}

by the malathion. By contrast, nigella oil especially attenuated malathion-induced histopathological effects in the liver. The neuroprotective effects of argan oil against malathion-induced neurotoxicity could be attributed to antioxidant properties of its bioactive compounds such as tocopherol, caffeic acid or oleuropein, whereas nigella oil exerted anti-inflammatory action through its main bioactive component thymoquinone (30-40\% approximately).

KEYWORDS: malathion, argan oil, nigella oil, memory, anxiety-like behavior, neuroprotection, hippocampus, prefrontal cortex.

\section{INTRODUCTION}

Malathion (O,O-dimethyl thiophosphate of diethyl mercaptosuccinate) is one of organophosphate (OP) insecticides still authorized throughout the world to prevent, control and eradicate pests. In the agricultural sector, it has a wide-spectrum effect against the pests of field crops, locusts and sucking insects of fruits and vegetables. For domestic as well as health and veterinary purposes, it demonstrates some efficient ectoparasiticide effects on both human and animal lice [1]. Malathion is considered as an OP with a moderate toxicity grade for humans and other mammals. However, because of the abuse 
of its use in agricultural practice in the United States of America, malathion is one of the major sources of occupational exposure to pesticides [2]. Also, malathion is well-known to exert its main action in pests by an irreversible inhibition of acetylcholinesterase (AChE) at the synaptic junction [3]. The first breakdown metabolite, malaoxon, is 40 times more toxic than malathion [4]. A previous research reported that exposure to malathion induced oxidative stress and lipid membrane peroxidation effects [5], whereas a few other studies found an inhibition of AChE in an experimental setting [6, 7]. Moreover, several researches using animal models have highlighted that very low level exposure to malathion altered brain development [8], changed liver and kidney histological structures [9], disrupted reproductive functions in males [10], and caused apoptotic neuronal death [11]. The delayed human health hazard effects of OPs including malathion are predominantly cognitive and behavioral deficits, and psychiatric disorders after chronic occupational exposure [12-14]. From these observations, the detrimental effects of malathion seem to be diverse. Most of the neurotoxicants cause the diseases by the neuroinflammatory and/or oxidative stress mechanisms; therefore an appropriate strategy to counteract those effects is drug-based antiflammatory or antioxidant intervention.

For this purpose, we promote the possible beneficial effects of both virgin Nigella sativa and Argania spinosa seed oils using an experimental model of malathion-induced neurotoxicity. Nigella sativa $L$ (Ranunculaceae), common name 'black cumin', grows in the Mediteranean area, especially in the north of Morocco and is well known as traditional medicine which is used in powder or oil forms against various diseases. In experimental studies, it was used to correct dyslipidemia and hyperglycemia [15], and also for its anti-inflammatory effect against rat's model of arthritis [16]. Argania spinosa L (Sapotaceae) is an endemic tree of Maghreb typically found in the south-west of Morocco. Argan oil is rich in antioxidant compounds and presents indescribable protective properties against obesity or human cardiovascular diseases $[17,18]$, anticonvulsant actions [19], as well as various nutritional and pharmacological positive effects [20]. However, to our knowledge, the effects of nigella and argan oils on the neurotoxicity caused by OP exposure have never been investigated. Hence, we examine the neuroprotective actions of the argan and nigella oil pretreatment on the cognitivebehavioral abilities using a rat's model of malathion-induced neurotoxicity, and specifically on biochemical and histopathological changes in brain.

\section{MATERIALS AND METHODS}

\subsection{Animals}

The experiment was carried out using 3-month-old male Wistar rats (175-200 g) locally obtained at the animal breeding house of Ibn Tofail University (Kenitra, Morocco). Animals were acclimated under controlled reference room temperature at $22-25^{\circ} \mathrm{C}$ with a good relative humidity (50-60\%), and submitted to a $12 \mathrm{H}$ light/dark cycle. They had free access to standard food (ALF SAHEL Company of Casablanca) and tap water. All experimental protocols were carried out according to NIH guide for the care and use of laboratory animals and approved by Ibn Tofail University Local Ethic Committee.

\subsection{Chemicals}

Malathion was commercial-grade (50\% purity), Ellman reagent (DTNB, Dithio-bis-nitrobenzoic Acid) and acetylthiocholine iodide were purchased (Sigma Aldrich, St Louis). Virgin argan and nigella oils were kindly donated.

\subsection{Animal treatments}

Animals were randomly assigned into four experimental groups $(n=7)$. Group 1 as vehicle received only $1 \mathrm{ml}$ of corn oil (Veh), Group 2 was given only $150 \mathrm{mg} / \mathrm{kg}$ (р.o.) malathion diluted in corn oil (Mal 150), Group 3 was pretreated orally with $2 \mathrm{ml} / \mathrm{kg}$ argan oil 1 hour before administration of malathion $150 \mathrm{mg} / \mathrm{kg}$ (р.o.) (Mal $150+$ Arg), Group 4 was pretreated orally with $1 \mathrm{ml} / \mathrm{kg}$ nigella oil and intoxicated 1 hour later with malathion 150 mg/kg (p.o.) (Mal 150 + Nig).

It was previously demonstrated that malathion at $150 \mathrm{mg} / \mathrm{kg}$ significantly impaired cognitivebehavioral capacities [6, 21]. Moreover, the effective dose of argan oil [22] and nigella sativa oil [23] was determined elsewhere. All the treatments lasted for 28 days because of the relative toxicity of nigella oil. 


\subsection{Behavioral testing}

\subsubsection{Open-field test}

The open-field apparatus is a square arena $(40 \mathrm{~cm}$ height, $100 \mathrm{~cm}$ width and $100 \mathrm{~cm}$ length) with the floor covered by a white consistent plastic. The floor was divided into 25 squares (5 rows of 5). The apparatus was illuminated under approximately 9lx. The animals were placed in the center and allowed 5 min to travel freely through the arena. A video tracker recorded the spontaneous locomotor activity, rearing frequency and grooming activity. Only the four paws being in the square was computed as a parameter of the square visited [24]. To avoid the clues related to the activity of the previous animal in the apparatus, we cleaned the apparatus with $10 \%$ of ethanol solution.

\subsubsection{Elevated plus maze (EPM)}

The anxiety level of rats was evaluated using EPM. The apparatus consists of four arms arranged in the form of a cross with the intersection as the common central platform $(5 \times 5-\mathrm{cm})$. There are two open arms (50-cm height $\mathrm{cm} \times 10$-cm width) opposed to two other arms enclosed by wooden walls (40-cm high). The EPM was raised $50 \mathrm{~cm}$ above the floor and lighted with a halogen lamp of 9lx. The test began by placing each animals onto the central square facing to the open arm and allowed 5 min to explore freely. The time spent in the open arms was computed to evaluate anxietyrelated behavior. The apparatus was cleaned with ethanol $10 \%$ between each test.

\subsubsection{Novel object recognition test (NORT)}

The NORT is suitable to assess AD-related cognitive impairment [25]. The object recognition test procedure was conducted as described by Enanceur and Delacour [26]. The apparatus is an open box with floor measurements $50 \mathrm{~cm}$ in length, $50 \mathrm{~cm}$ in width, and $40 \mathrm{~cm}$ in height. In the trial session (familiarization phase), rats were allowed 5 min to freely explore the box with two identical objects (familiar object) and were returned to their home cage. After $2 \mathrm{~h}$, to evaluate short-term recognition memory (STM), rats were placed back in the open field in which one familiar object was replaced by another different with respect to the size, the color and the shape. Then, animals were again allowed 5 min to explore the box with the novel and the familiar objects. Finally, to evaluate long-term recognition memory (LTM) $24 \mathrm{~h}$ after the familiarization phase, rats were allowed to explore again two objects for $5 \mathrm{~min}$, a familiar one and another novel object different to that of short-tem phase. The objects and the arena were cleaned with ethanol $70 \%$ during the intertrial period. The exploration time of each object was recorded using a video tracker. The ratio of the preference for the novel object was calculated from the frequency of exploration of the novel object divided by the total frequency for exploring both novel and familiar objects. Exploration behavior' is defined as 'the rat directing its nose at a distance equal to or less than $3 \mathrm{~cm}$ from the object without touching it.

\subsection{AChE inhibition assay}

After behavioral tests, rats were anesthezied with chroral hydrate (400 mg/kg, i.p) and killed by decapitation. Brain areas related to the hippocampus, prefrontal cortex (PFC) and cerebellum were removed and homogenized in ice-cold $50 \mathrm{mM}$ Tris-HCl buffer ( $\mathrm{pH}$ 7.4). The homogenate was then centrifuged at $3000 \mathrm{rpm}$ for $30 \mathrm{~min}$ at $4^{\circ} \mathrm{C}$ to obtain a supernatant. AChE activity was assayed according the method of Ellman [27] using acetylthiocholine iodide as substrate. The reaction mixture contained $100 \mu \mathrm{L}$ of supernatant, $150 \mu \mathrm{L}$ of DTNB (100 mM), $2.8 \mathrm{~mL}$ of phosphate buffer (100 mM, pH 8), and $50 \mu \mathrm{l}$ of substrate $(75 \mathrm{mM})$. The acetylthiocholine iodide is hydrolyzed by the AChE enzyme in the sample leading to the release of thiocholine. Then, the thiol group of thiocholine is reduced by the DTNB to form TNB with a dark yellow color. The absorbance was measured at $412 \mathrm{~nm}$ for $10 \mathrm{~min}$.

\subsection{Histological study}

The tissues were emmbebed in paraffin wax and histostained with haematoxylin and eosin using the method described by [28] with some light modifications. Beforehand, the brain and liver were fixed in formol $10 \%$. Then, tissues inside the cassette were removed from formol and dehydrated with a gradual bath of ethanol concentrated at $70 \%$ (soaked two times for $60 \mathrm{~min}$ ), then with one at $90 \%$ (soaked two times for $60 \mathrm{~min}$ ) and finished with toluene (washed two times for $60 \mathrm{~min}$ ). Thenceforth, tissues were embedded with paraffin wax and cut using microtome (3- $\mu \mathrm{m}$ sections). Brain 
sections correspondent to gyrus dentate (GD) and cornu ammonis (CA1) of hippocampus and PFC, and the liver sections were spread out on slides. The next step consisted of dewaxing under a stove for 3 hours. The tissue sections were rehydrated with toluene solution followed by successive baths of ethanol solution (concentrated from 100 to $70 \%$ ). After a rinse with distilled water, the sections were stained with hematoxylin and eosin ( $\mathrm{H} \& \mathrm{E})$. The well-stained slides were mounted with a coverslip and the images were captured using light microscope connected to a 5.0 MP Amscope camera.

\subsection{Data analysis}

GraphPad Prism 6.0 version was used to record data for their analysis. The experimental data results were expressed as mean \pm S.E.M (standard error of mean). Statistical differences between the groups were determined by one-way ANOVA followed by Tukey post-hoc test for multiple comparisons. $P<0.05$ was considered statistically significant.

\section{RESULTS}

\subsection{General parameters and body weight gain}

We found no sign of systemic toxicity in the form of salivation, convulsion, tremors, and ptosis among others in any of the experimental groups. The body weight gain was not significantly changed among the studied groups over the 4 weeks of treatment (data not shown).

\subsection{Effects of argan and nigella oils on malathion-induced cognitive-behavioral changes}

\subsubsection{Spontanous locomotor and exploratory activities in open-field test}

As shown in the Fig. 1A, no significant changes were found regarding basal locomotor activity since the spontaneous locmotor activity had not changed between experimental groups $(P=0.58)$. The malathion significantly impaired the exploratory behavior which is reflected by a significant reduction in the number of rearings when compared to other studied groups $(P=0.009)$. Post-hoc analysis had revealed that argan oil pretreatment significantly increased the number of rearings $(P<0.05$ vs. Mal 150 group of rats) incomparable to the results of nigella oil ( $P>0.05$ vs. Mal 150 group of rats) (Fig. 1B). The grooming number varied significantly among the treated groups $(P=0.004)$. The argan oil pretreatment had significantly prevented malathioninduced alteration in grooming behavior $(P<0.01$ vs. Mal 150) compared to nigella oil $(P<0.05$ vs. Mal 150). In addition, the pretreatment of the malathion rats with argan oil caused significant improvement in grooming behavior when compared to that with nigella oil $(P<0.05)$ (Fig. 1C).

\subsubsection{Anxiety-like behavior in EPM}

The administration of malathion caused an anxietylike behavior in the rats which was expressed by a significant lessening of the time spent in open arm $(P<0.05)$. However, the pretreatment with both argan and nigella oils mitigated anxiety-like behavior associated with an increase in the time spent in the open arm of EPM (Fig. 2).

\subsubsection{Recognition memory in NORT}

The short-term recognition index was different among the studied groups $(P<0.001)$. The posthoc analysis revealed that the lowest recognition index was found in malathion rats. By contrast, the pretreatment with argan oil significantly prevented the alteration of short-term recognition memory abilities induced by malathion $(P<0.001$ vs. malathion rats), but the pretreatment with nigella oil did not have the same effects $(P>0.05$ vs. malathion rats) (Fig. 3A).

The malathion rats also displayed an impairment of long-term recognition memory, which was expressed by a significant reduction of recognition index $(P<0.001)$, when compared to those pretreated with argan oil. The pretreatment with nigella oil didn't improve the altered long-term recognition memory in the malathion rats $(P>0.05)$. Interestingly, there is high significant difference regarding long-term recognition index between the argan oil and nigella oil pretreatments $(P<$ 0.001) (Fig. 3B).

\subsection{Effects of argan and nigella oils on malathion-induced AChE inhibition}

The Fig. 4 indicates the effects of treatments on AChE inhibition rate.

In the hippocampus, the rates of AChE inhibition significantly varied among the animals treated $(P$ $<0.001)$. The exposure to malathion had increased to $+232 \%$ the rate of AChE inhibition from baseline value of Veh group $(P<0.001)$. The pretreatment 

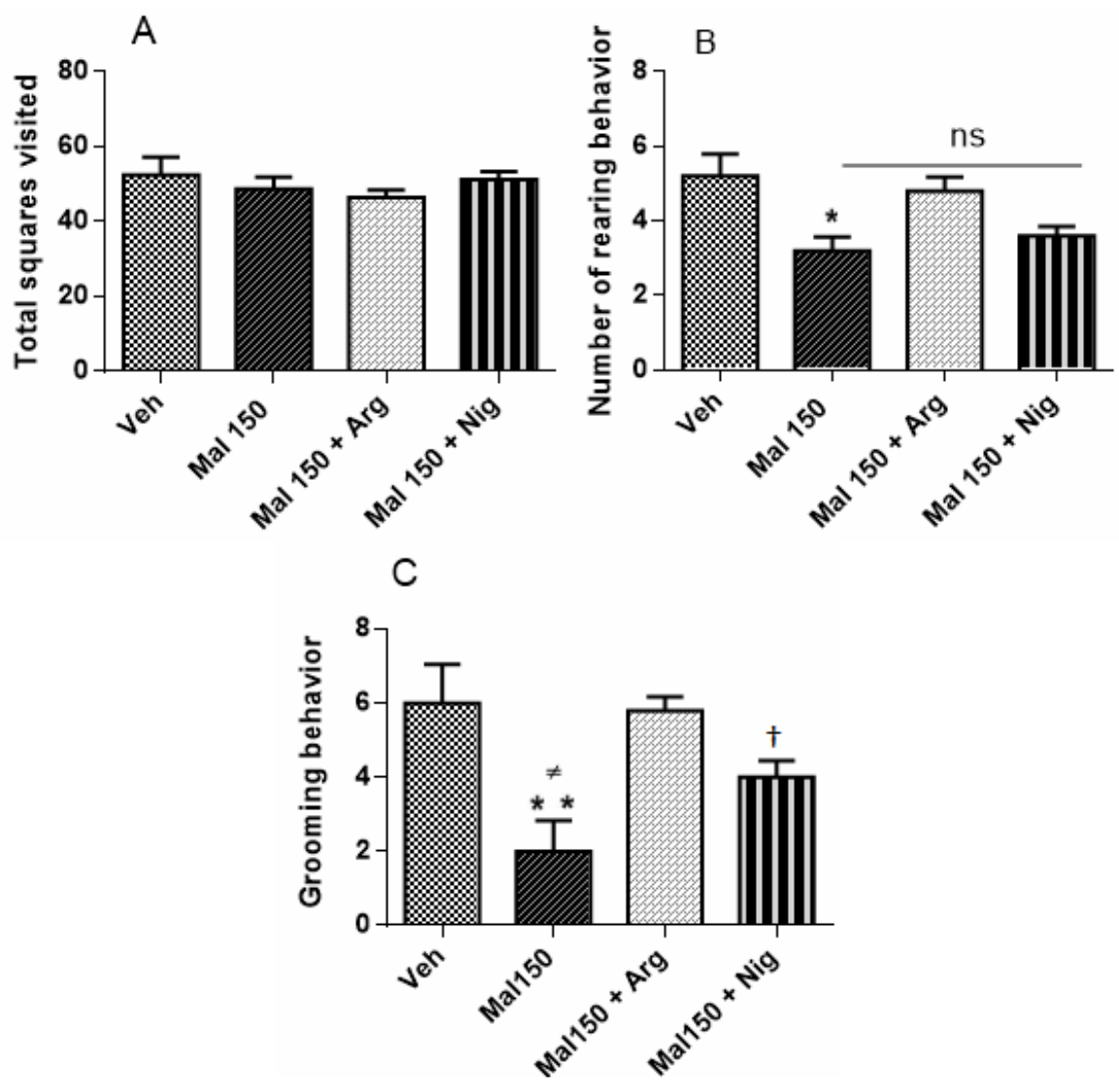

Fig. 1. Effects of argan and nigella oil pretreatments on behavior in rat's model of malathion-induced neurotoxicity. A, Locomotor activity; B, Exploratory activity and C, Grooming behavior. Data are represented as mean \pm SEM. One-way ANOVA/Tukey post hoc test. ${ }^{*} P<0.05$ (Mal 150 vs. Veh) ; ${ }^{* *} P<0.01$ (Mal 150 vs. Veh or Mal $150+$ Arg) ; ${ }^{\neq} P<0.05$ (Mal 150 vs. Mal $150+$ Nig) ${ }^{\dagger} P<0.05$ ( Mal $150+$ Nig vs. Mal $150+$ Arg or Veh). ns, non significant.

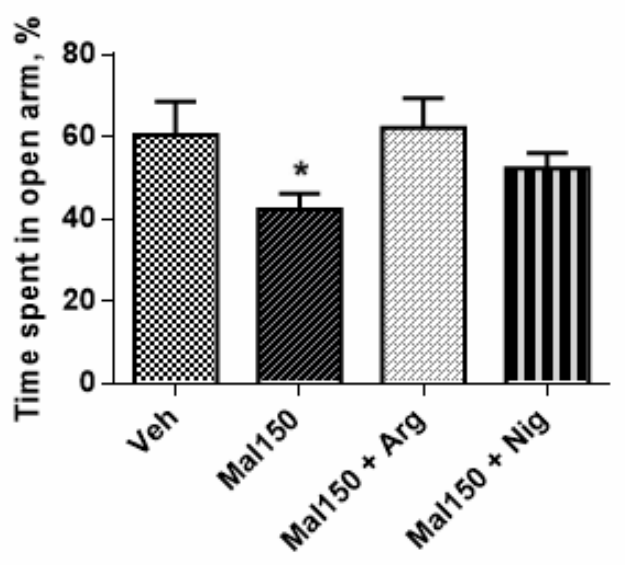

Fig. 2. Effects of argan and nigella oil pretreatments on anxiety-like behavior level in rat's model of malathioninduced neurotoxicity. Data are represented as mean \pm SEM. One-way ANOVA/Tukey post hoc test. * $P<0.05$ (Mal 150 vs. other experimental groups). of malathion rats with nigella oil significantly prevented the rate of AChE inhibition, $+4 \%$ only $(P<0.001$ vs. malathion rats). By contrast, the pretreatment with argan oil didn't significantly prevent malathion-indced inhibition of AChE, $+123 \%$ of inhibition rate ( $P>0.05$ vs. malathion rats). Finally, the nigella oil pretreatment showed beneficial effects compared to argan oil pretreatment with regard to the prevention of malathion-induced AChE inhibition $(P<0.05)$

In the PFC, we also found highly significant differences of the AChE inhibition rates among the experimental groups $(P<0.001)$. The pretreatment of malathion rats with nigella oil maintained the AChE inhition rate at the baseline value $+0 \%(P<0.001$ vs malathion rats), which is incomparable to the pretreatment with argan oil +156 ( $P<0.05$ vs malathion rats). 

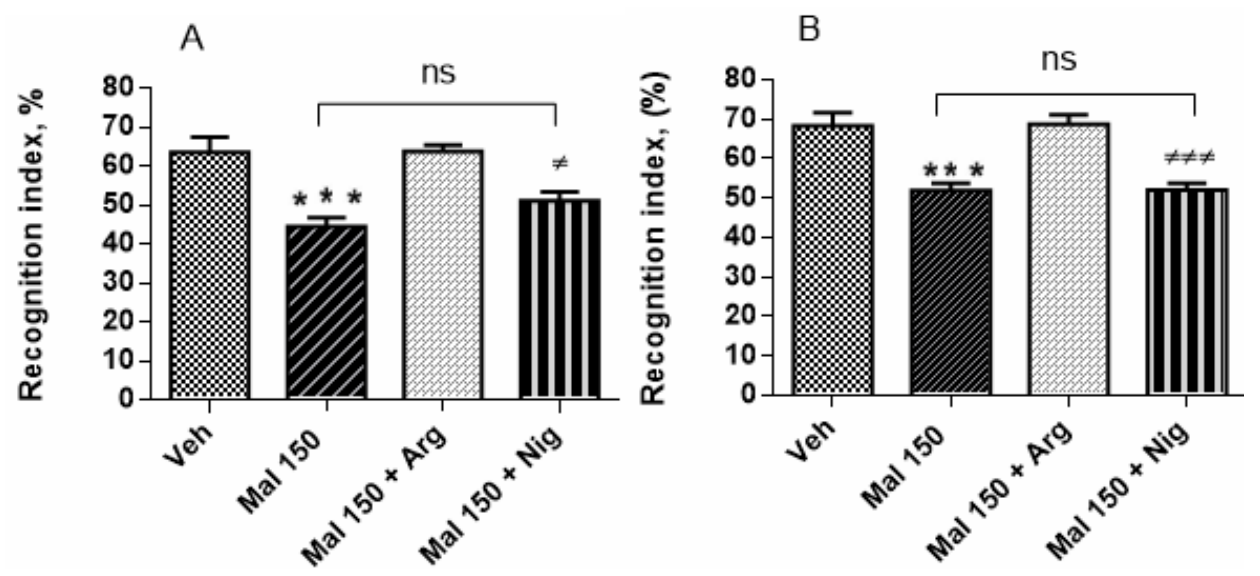

Fig. 3. Effects of argan and nigella oil pretreatments on recognition memory abilities in rat's model of malathioninduced neurotoxicity. A, Short-term recognition memory; B, Long-term recognition memory. Data are represented as mean \pm SEM. Data are represented as mean \pm SEM. One-way ANOVA/Tukey post hoc test. ${ }^{* * *} P<0.001$ (vs. Veh and Mal $150+$ Arg) $;{ }^{\neq} P<0.05,{ }^{\#} P<0.001$ (Mal $150+$ Arg vs. Mal $150+$ Nig); ns, non -significant.


Fig. 4. Effects of argan and nigella oil treatments on AChE inhibition rate in rat's model of malathion-induced neurotoxicity. Data are represented as mean \pm SEM. One-way ANOVA/Tukey post hoc test. $* * * P<0.001$ (Mal 150 vs. Veh or Mal + Nig) $;{ }^{\neq} P<0.05$ (Mal $150+$ Arg vs. Veh or Mal $150+$ Nig) $;{ }^{\dagger} P<0.05$ (Mal 150 vs. Mal $150+$ Arg); ns, non significant. 
In the cerebellum, both argan and nigella oil pretreatments significantly mitigated the effects of malathion-induced AChE inhibition $(P<0.001)$.

\subsection{Histological analysis}

\subsubsection{Histopathological analysis of the liver sections}

The effects of the treatments on the liver tissue are presented in the Fig. 5. We noted in the Fig. 5A, a compact structure with normal shape of hepatocytes, no fibrosis as well as the portal system at the right place (yellow arrow). We observed in malathion rats a hepatocytic necrosis (blue arrow), an enlargement of the portal space with fibrosis (yellow arrow), and congestion of blood vessel with hemorrhage (green arrow) (Fig. 5B). Similar observations were made in the malathion rats pretreated with argan oil but a lesser degree (Fig. 5C). By contrast, a reduction of enlargement portal space (yellow arrow) and fibrosis were observed in malathion rats pretreated with nigella oil (Fig. 5D).

\subsubsection{Histopathological analysis in the PFC sections}

From the PFC sections, we observed healthy tissues in Veh rats (Fig. 6A), while the exposure to malathion caused an inflammation marked by cytoplasmic eosinophilia condensation (green arrow), vascular congestion (blue arrow) giving rise to erythrocytes extravasation (black arrow), and neuronal depopulation (Fig. 6B). However, the pretreatment of malathion rats with argan oil prevented histological changes (Fig. 6C). The malathion rats pretreated with nigella had a PFC with hypereosinophilia, but the neuronal loss was less pronounced compared to malathion rats.

\subsubsection{Histological changes in the hippocampal tissue}

The Fig. 7 shows a panoramic view of the organization of hippocampus cell layers. In Veh rats, the arrangement of the pyramidal cells of hippocampal CA1 subfield and granule cells of gyrus dentate (GD) were well-defined. In malathion rats, we noted a neuronal loss in GD layer (black arrow), while the CA1 cellular layers were dislocated. However, the pretreatment of malathion rats with argan oil or nigella oil showed beneficial effects on the GD and and CA1 cell layer organization, except for the presence of some clear halo (blue arrow) and some slight vascular congestion (yellow arrow).

\section{DISCUSSION}

Several studies were carried out to assess the effects of malathion toxicity on health using both in vivo and in vitro experimental models. For instance, the malathion-induced cytoxicity and genotoxicity effects included the appearance of human carcinoma liver cells [29]. In the animal model, numerous studies have reported that a chronic exposure to malathion caused reproductive, liver and kidney function disruptions as well as metabolic and biochemical disorders [10, 30, 31]. Some other studies have highlighted the toxicity link between the malathion exposure and cognitive impairment and/or also affective disorders such as anxiety- or depressive-like behavior [6, 7, 21, 32]. By contrast, there is a scarcity of researches which have focused on the neuroprotective actions of natural products against malathion-induced neurotoxicity associated to subsequent cognitivebehavioral effects, and also on histopathological implications. This present work aimed to explore for the first time whether both moroccan traditional argan and nigella oils could be used as natural drug substances to prevent adverse effects of malathion on the brain.

Based on our results, we confirmed the detrimental effects of malathion on the brain and behavior. The group of rats exposed to malathion alone for 28 days (150 mg/kg p.o.) expressed a general hypoactivity in open-field apparatus and an anxiety-like behavior in EPM, as well as shortterm and long-term recognition memory deficits. Moreover, we found a significant inhibition rate of AChE in hippocampus, PFC and cerebellum areas. An analysis of the liver tissue sections revealed some necrosis and fibriosis while hippocampal and PFC tissues showed some inflammation, neurodegeneration and cytoarchitecture disorganization. Our results are in accordance with those of previous researches aforementioned. There are two supposed physiopathological mechanisms by which malathion induces neurotoxicity, namely, AChE inhibition [6, 33, 34] and oxidative stress process [29, 35, 36]. In fact, OP compounds including malathion exert toxicity by inhibiting AChE through phosphorylation of its active seric amino acid site [37]. The dysregulation of cholinergic system resulting from AChE inhibition is responsible for several 

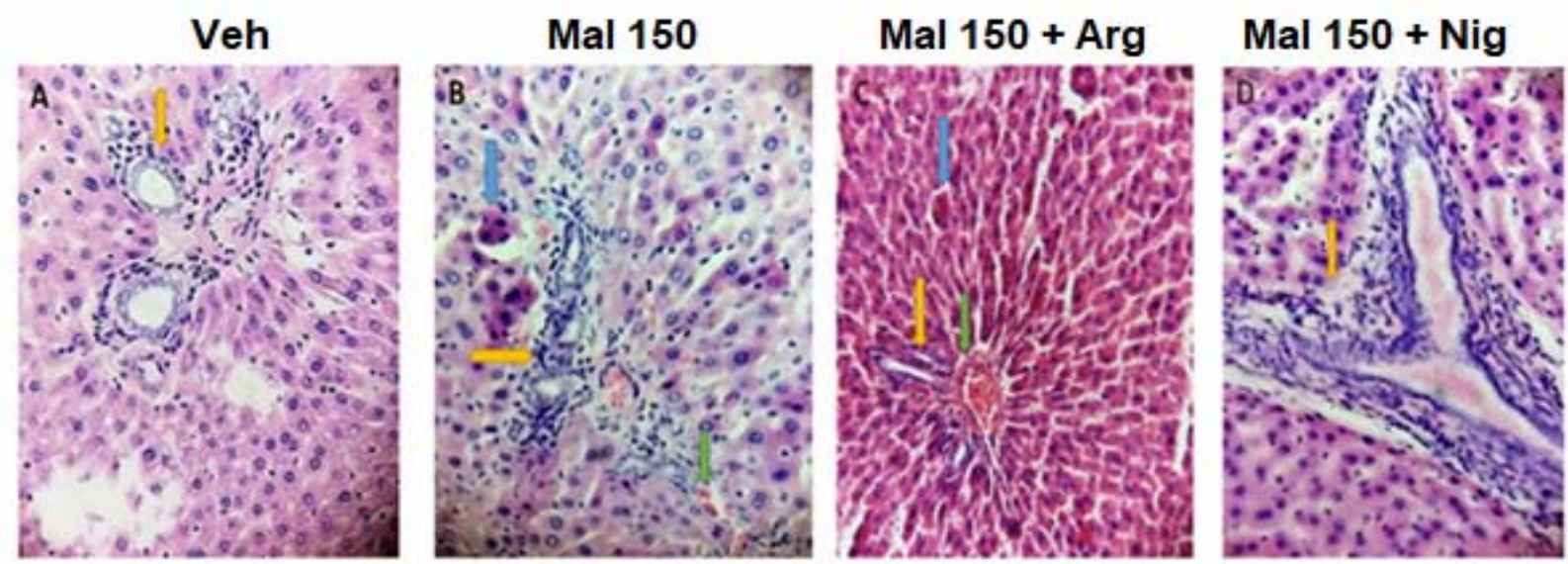

Fig. 5. Effects of argan and nigella oil pretreatments on malathion-induced histopathological changes in the liver (H \& E staining, $100 \mu \mathrm{m}$ scale bar).
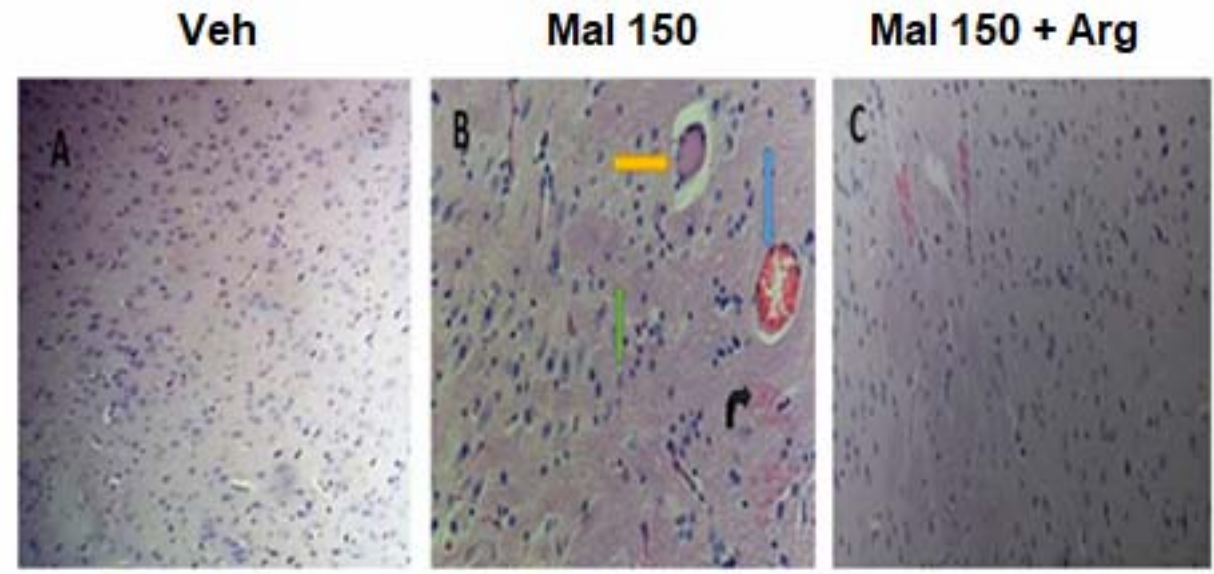

Mal $150+$ Nig

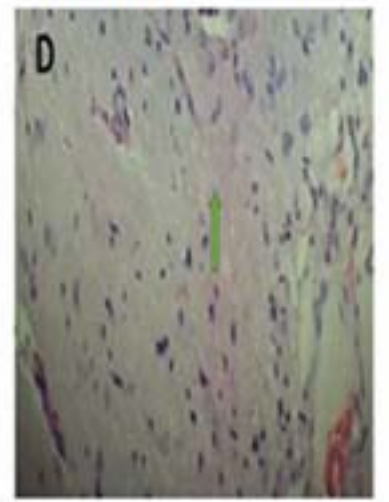

Fig. 6. Effects of argan and nigella oil pretreatments on malathion-induced histopathological changes in the PFC (H \& E staining, $100 \mu \mathrm{m}$ scale bar).

physiological and neurological disorders including mood and cognitive disorders [38, 39]. Clearly, Acker et al. [33] have shown positive correlation between the AChE inhibition by malathion (200 $\mathrm{mg} / \mathrm{kg}$ p.o.) during brain development and various neurobehavioral impairments.

Those authors have also reported that malathion inhibits $\mathrm{Na}^{+} / \mathrm{K}^{+}$ATPase activity in the cerebral cortex of adult rats, and that could alter the neuronal membrane excitability and nerve impulse transmission processes [40]. Moreover, malathion caused oxidative stress by depleting the natural antioxidants and by increasing the lipid membrane peroxidation in cortice cell medium [41], as well as in brain tissue when administrated to rats at
$200 \mathrm{mg} / \mathrm{kg}$ [5]. Apparently, that could be due to an increase in reactive oxygen species (ROS). In fact, the hepatic mitochondrial metabolism of OP compounds (including malathion) is suggested to be the key mechanism to change antioxidant defence systems and damage cells and tissues via membrane lipid peroxidation and free radical generation [42, 43]. One of the subsequent effects of malathioninduced neurotoxicity is neuronal apoptic death as observed when studying the neurodegeneration model of Alzheimer's disease [11]. Taken together, the findings of these researches could further elucidate mechanisms of the neuronal loss in PFC, and in hippocampal CA1 and DG subfields observed in the group of malathion rats of our experiment. 

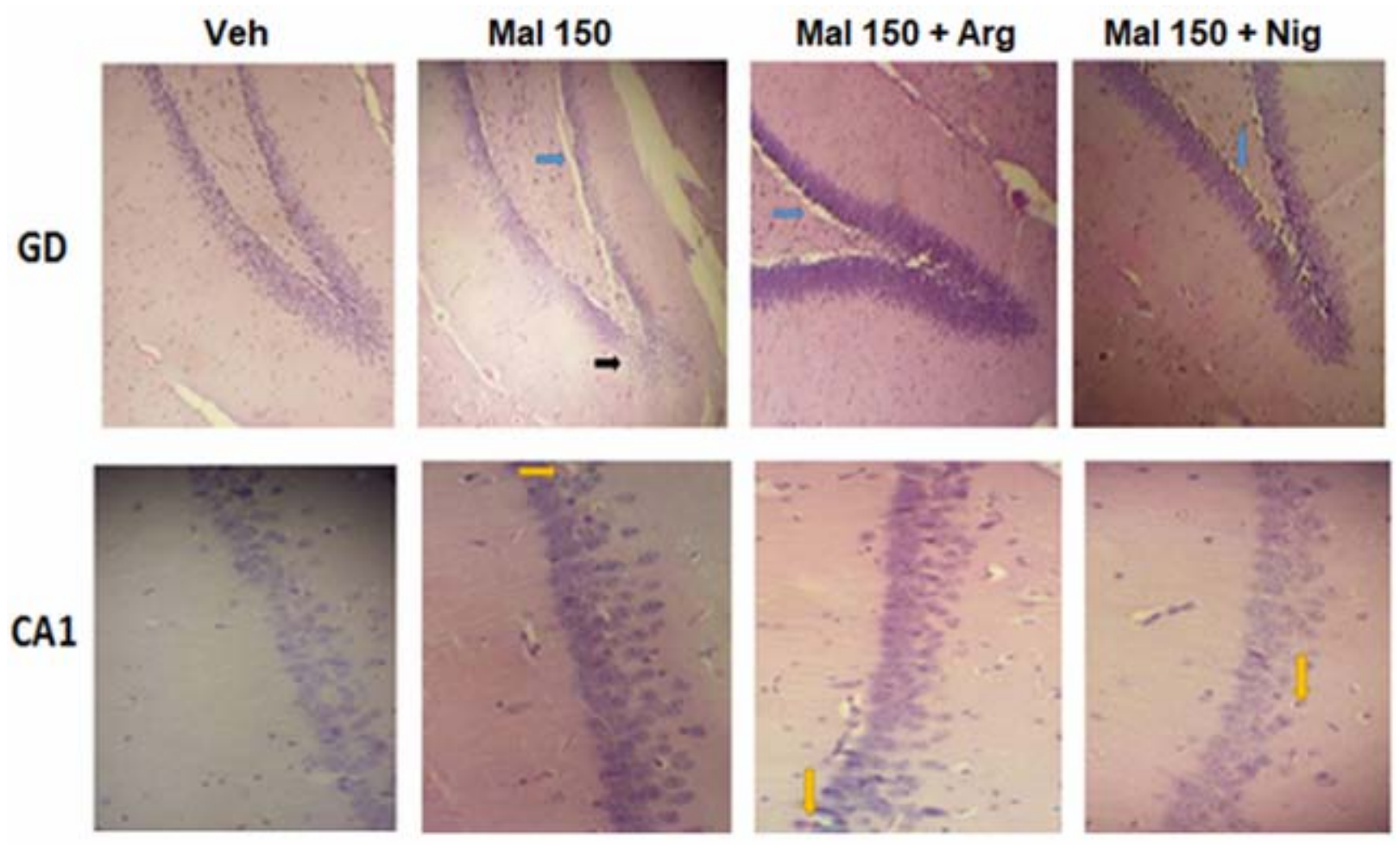

Fig. 7. Effects of argan and nigella oil pretreatments on malathion-induced histopathological changes in hippocampal areas. GD, gyrus dentate; CA1, Conu ammornus (H \& E staining, $100 \mu \mathrm{m}$ scale bar).

The pretreatment of malathion group of rats with natural products, either argan oil or nigella oil, improved the altered cognitive-behavioral abilities in malathion rats. Additionally, argan oil protected against cerebral tissue alteration and neurodegeneration mainly in hippocampus and the PFC, whereas nigella oil predominantly prevented AChE inhibition. We suggest that the neuroprotective actions of virgin argan oil against malathion-induced neurodegeneration could be attributed to antioxidant compounds such as tocopherols ( $600 \mathrm{mg} / \mathrm{kg}$ of total chemical components of the oil), and other polyphenols such as caffeic acid and oleuropein [44-46]. The tocopherols are antioxidant compounds well-known as potent free radical scavengers and quenchers of singlet oxygen. For instance, it has been reported that $\alpha$ tocopherol protected tissues against oxidative damage, it also has been demonstrated some antiinflammatory properties [47]. A diet supplemented with $\alpha$-tocopherol is capable of promoting an oxydo-reduction of the organic free radicals (ROO), and an inhibition of membrane lipid peroxidation process. Its anti- inflammatory effects are a result of the actions on various enzymes involved in the inflammation pathways such as phospholipase A2, cyclooxygenase 2, and protein kinases among others [47]. In addition, the caffeic acid has displayed some antioxidant effects against iron-induced free radical generation [48] while oleuropein induces some neuroprotective effects based on their antioxidant and anti-inflammatory properties [49]. Akbel et al. [5] have proven the protective effects of a polyphenol compound resveratrol against malathion-induced oxidative stress, lipid peroxidation as well as its ability to prevent AChE inhibition in the brain of rats. More importantly, those authors reported that the antioxidant resveratrol changed the level the level of 8-OHdG in a dose-dependent manner and protected brain tissue from mutagenic damage and neuronal degeneration caused by malathion [5]. In the present study, we found some attenuation of inflammation signs such as hepatic necrosis and congestion of blood vessels with hemorraghe in malathion group of rats pretreated with nigella oil. Our results are in agreement with those of $\mathrm{Al}-$ Seeni et al. [23] who have highlighted the 
hepatoprotective activity of nigella oil on the toxicant CCl4 which damages liver cells by inflammation. On the other hand, a previous research indicated that nigella sativa oil significantly prevented inflammation using a rat's model of human rumathoid arthritis, by reducing specific molecular marker levels over a 25-day period of treatment [16]. Those beneficial effects of nigella sativa oil could be attributed to its main bioactive compound thymoquinone, which has multiple pharmacological actions including anti-inflammatory properties [50, 51]. Importantly, it was demonstrated that thymoquinone treatment of malathion-inhaled rats remarkably decreased hepatic tisue injury [52], as well as protected liver cells against diazinon (an OP with high toxicity grade)-induced hepatotoxicity [53]. Also, nigella oil could have some antioxidant vertus based on the $\alpha$ - and $\gamma$ tocopherols components [54]. This property could explain the non-neurodegeneration observed in hippocampal GD area of malathion rats pretreated with nigella oil. However, it needs to be further appreciated by studies based on its dose-dependent effects because of relatively its low content of $\gamma$ tocopherol (103 $\mathrm{mg} / \mathrm{kg}$ ) and $\alpha$-tocopherol (26 $\mathrm{mg} / \mathrm{kg}$ ), when compared to argan oil tocopherol concentration (600 mg/kg).

\section{CONCLUSION}

This study using an animal model has confirmed the deleterious effects of sub-chronic exposure to malathion on the brain. The pretreatment with argan oil has significantly mitigated malathioninduced memory deficits and anxiety-like behavior, as well as prevented hippocampal and PFC neuronal loss. By contrast, the pretreatment of malathion rats with nigella oil predominantly had a hepatoprotective effect. Those beneficial effects are suggested to be related to the antioxidant properties of tocopherol contained in the argan oil, but also due to the thymoquinone from nigella oil, which has some anti-inflammatory effects. The combination of both argan and nigella oils should strengthen the neuroprotective actions against malathion-induced neurotoxicity.

\section{ACKNOWLEDGEMENTS}

The authors acknowledge Professeur TLIGUI Noursaid from the Anatomy and Pathology
Laboratory of the Agronomy and Veterinary Institute (Rabat), for the assistance during the histological analysis.

\section{ABBREVIATIONS}

CCl4, Carbon tetrachloride; OHdG, Hydroxydeoxyguanosine; TNB, 2-nitro-5thiobenzoic acid.

\section{CONFLICT OF INTEREST STATEMENT}

The authors declare no conflict of interest.

\section{REFERENCES}

1. Maroni, M., Colosio, C., Ferioli A. and Fait, A. 2000, Toxicology, 7, 1-118.

2. Bonner, M., Coble, J., Blair, A., Freeman, L., Hoppin, J., Sandler, D. and Alavanja, M. 2007, Am. J. Epidemiol., 166, 1023-1034.

3. Cabello, G., Valenzuela, M., Vilaxa, A., Durán, I. R., Hrepic, N. and Calaf, G. 2001, Environmental Health Perspectives, 109(5), 471-479.

4. $\quad$ Aldridge, W. N., Miles, J. W., Mount, D. L. and Verschoyle, D. R. 1979, Archives of Toxicology., 42 (2), 95-106.

5. Akbel, E., Arslan-Acaroz, D., Demirel, H. H., Kucukkurt, I. and Ince, S. 2018, Toxicology Research., 7(3), 503-512.

6. N’Go, P. K., Azzaoui, F-Z., Ahami, A. O. T., Soro, P. R., Najimi, M. and Chigr, F. 2013a, Health, 5(3A), 603-611.

7. Assini, F. L., Zanette, K. D., Brocardo, P. S., Pandolfo, P., Rodrigues A. L. S. and Takahashi, R. N. 2005, Environmental Toxicology and Pharmacology, 20, 443-449.

8. Richendrfer, H. and Creton, R. 2015, Neurotoxicology, 49, 50-58.

9. Al-Attar, A. M. 2010, Journal of Biomedicine and Biotechnology, 1-8.

10. Selmi, S., Rtibi, K., Grami, D., Sebai, H. and Marzouki, L. 2018, Lipids in Health and Disease, 17(1).

11. Venkatesan, R., Park, Y. U., Ji, E., Yeo, E-J. and Kim, S. Y. 2017, Cell Death Discovery, 3, 17007.

12. Nigg, H. N. and Knaak, J. B. 2000, Reviews of Environmental Contamination \& Toxicology, 163, 29-111. 
13. Kamel, F. and Hoppin, J. A. 2004, Environ. Health Perspect, 112, 950-958.

14. Tanner, C. M., Goldman, S. M., Ross, G. W and Grate, S. J. 2014, Alzheimer Dement., 2002(10), 213-225.

15. Zaoui, A., Cherrah, Y., Alaoui, K., Mahassine, N., Amarouch, H. and Hassar, M. 2002, Journal of Ethnopharmacology, 79, 23-26.

16. Nasuti, C., Fedeli, D., Bordoni, L., Piangerelli, M., Servili, M., Selvaggini, R. and Gabbianelli, R. 2019, Antioxidants, 8(9), 342.

17. Sour, S., Belarbi, M., Sari, N., Benammar, C. H., Baghdad, C. H. and Visioli, F. 2015, Nutrition, Metabolism \& Cardiovascular Diseases., XX, 1-6.

18. Sour, S., Belarbi, M., Khaldi, D., Benmansour, N., Sari, N., Nani, A., Chemat, F. and Visioli, F. 2012, Br. J. Nutr., 107, 1800-5.

19. Bahbiti, Y., Ammouri, H., Berkiks. I., El Hessni, A., Ouichou, A., Nakache, R., Chakit, M., Bikjdaouene, L. and Mesfioui, A. 2016, Nutritional Neuroscience, 21(2), 1-7.

20. El Abbassi, A., Khalid, N., Zbakh, H. and Ahmad, A. 2014, Critical Reviews in Food Science and Nutrition, 54(11), 1401-1414.

21. N’Go, P, K., Azzaoui, F-Z., Soro, P. R., Najimi, M., Chigr, F., Samih, M., Ahami, A. O. T. and Chigr, F. 2013b, JBBS., 3, 331-340.

22. Bellahcen, S., Mekhfi, H., Ziyyat, A., Legssyer, A., Hakkou, A., Aziz, M. and Bnouham, M. 2012, Phytother. Res., 26, 180 $-185$.

23. Al-Seeni, M. N., El Rabey, H. A., Zamzami, M. A. and Alnefayee, A. M. 2016, Complementary and Alternative Medicine, 16, 438.

24. Walsh, R. N. and Cummins, R. A. 1976, Psychol. Bull., 83, 482-504.

25. Bryan, K. J., Lee, H., Perry, G., Smith, M. A. and Casadesus, G. 2009, J. J. Buccafusco (Ed.), 1-18.

26. Ennaceur, A. and Delacour, J. 1988, Behav. Brain Res., 31, 47-59.

27. Ellman, G. L. 1959, Arch. Biochem. Biophys., 82, 70-77.

28. Fischer, A. H., Jacobson, K. A., Rose. J. and Zeller, R. 2005, Hematoxylin and eosin (H \& E) staining. CSH Protoc., 2008.

29. Moore, P. D., Yedjou, C. G. and Tchounwou, P. B. 2010, Environ. Toxicol., 25, 221-226.
30. Selmi, S., Jallouli, M., Gharbi, N. and Marzouki, L. 2015, J. Med. Food., 18(10), 1103-1111.

31. Pournourmohammadi, S., Farzami, B., Ostad, S. N., Azizi, E. and Abdollahi, M. 2005, Environmental Toxicology and Pharmacology, 19 (1), 191-196.

32. Brocardo, P. S., Assini, F., Franco, J. L., Pandolfo, P., Müller, Y. M. R., Takahashi, R. N., Alcir, L. D. and Ana, L. S. R. 2007, Toxicological Sciences, 97(1), 140-148.

33. Acker, C. I., Souza, A. C., Pinton, S., Da Rocha, J. T., Friggi, C. A., Zanella, R and Nogueira, C. W. 2011, Ecotoxicol. Environ. Saf., 74 (8), 2310-2315.

34. Selmi, S., El-Fazaa, S. and Gharbi, N. 2012, Environ. Toxicol. Pharmacol., 34(3), 753-760.

35. Wankhade, V. W. 2012, RJET., 6(4), 142.

36. Fortunato, J. J., Agostinho, F. R., Réus, G. Z., Petronilho, F. C., Dal-Pizzol, F. and Quevedo, J. 2006, Neurotox Res., 9(1), 23-28.

37. Wang, L. M., Ye, W. H., Zhou, S. S., Lin, K. D., Zhao, M. R. and Liu, W. P. 2009, J. Environ. Sci. Health B., 44, 38-43.

38. Dulawa, S. C. and Janowsky, D. S. 2019, Molecular Psychiatry, 24(5), 694-709.

39. Ouagazzal, A. M., Kenny, P. J. and File, S. E. 1999, Psychopharmacology,144, 54.

40. Acker, C. I., Luchese, C., Prigol. M. and Nogueira, C. W. 2009, Neurosci. Lett., 455, 168-172.

41. Salama, M., Lotfy, A., Fathy, K., Makar, M., El-emam, M., El-gamal, A., Badawy, A., Wael, M. Y. M and Sobh, M. 2015, Applied \& Translational Genomics., 7, 13-18.

42. Valdiglesias,V., Pásaro, E., Méndez, J. and Laffon, B. 2010, Arch Toxicol., 84, 337-51.

43. Altuntas, I., Kilinc. I., Orhan, H., Demirel, R., Koylu, H. and Delibas, N. 2004, Hum Exp Toxicol., 23, 9-13.

44. Aguilera, C. M., Mesa, M. D., RamirezTortosa, M. C., Nestares, M. T., Ros, E., and Gil, A. 2004, Clin. Nutr., 23, 673-681.

45. Khallouki, F., Younos, C., Soulimani, R., Oster, T., Charrouf, Z., Spieglehalder, B. Batsch, H. and Owen, R. W. 2003, Eur. J. cancer prev.,12, 67-75.

46. Chimi, H., Rahmani, M. and Cillard. P. 1988, Actes Inst. Agron. Vét, 17-21.

47. Jiang, Q. 2014, Biology \& Medicine, 72, 7690. 
48. Genaro-Mattos, T. C., Maurício, Â. Q., Rettori, D., Alonso, A. and Hermes-Lima, M. 2015, A Chemical Approach PLoS One, 10(6), e0129963.

49. Sun, W., Frost, B. and Liu, J. 2017, Oncotarget, 8(11), 17409-17409.

50. Gholamnezhad, Z., Havakhah, S. and Boskabady, M. H. 2016, J. Ethnopharmacol., 190, 372-386.

51. Ikhsan, M., Hiedayati, N., Maeyama, K. and Nurwidya, F. 2018, BMC Res. Notes, 11, 744.
52. Abdo, W., Elmadawy, M. A., Abdelhiee, E. Y., Abdel-Kareem, M. A., Farag, A., Aboubakr, M., Ghazy, E. and Fadl, S. E. 2021, Scientific Reports., 11, 2498.

53. Ghazy, E., Mokh, A., Abdelhady, D., Goda, W. and Hashem, E. 2019, Slov. Vet. Res., 56(22 Suppl.).

54. Gharby, S., Harhar, H., Guillaume, D., Roudani, A., Boulbaroud, S., Ibrahimi, M., Ahmad M., Sultana, S., Ben Haddah, T., Chafchaouni-Moussaoui, I. and Charrouf, Z. 2015, J. Saudi Soc. Agric. Sci., 14, 172-177. 